\title{
Utilización de implantes de sillicona en la enfermedad de Dupuytren
}

\author{
Use of silicone implants \\ in Dupuytren's disease
}

\author{
Ballesta Alcaraz, J.*, Ros Cantó, V.**, Liebana Pérez, C.***, Torres Maczassek, A.***
}

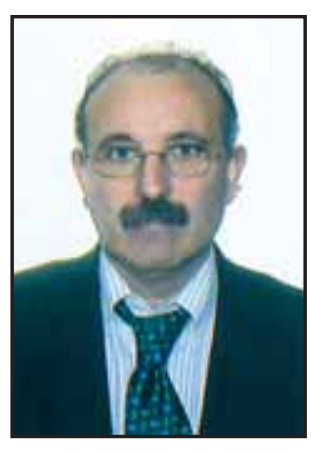

Ballesta Alcaraz, J.

\section{Resumen}

Presentamos nuestra experiencia en el tratamiento de la enfermedad de Dupuytren mediante la utilización de unos implantes de silicona que se colocan en la palma de la mano una vez efectuada la aponeurectomia. Efectuamos este tratamiento en un grupo de 44 pacientes afectos de dicha enfermedad a los que hemos aplicado unos criterios de selección tales como el grado de afectación y que no fueran manos ya intervenidas con anterioridad. La idea es presentar una técnica quirúrgica que soluciona de una manera eficaz un determinado tipo de casos. La tolerancia y resultados dan validez a la técnica.
Palahras clave Enfermedad de Dupuytren. Implantes de silicona. Aponeurectomía.

Código numérico 331, 1586

\section{Key words Dupuytren dissease. Silicone implant. Aponeurectomy.}

Código numérico 331, 1586 
Historia

El conocimiento de la contractura de la aponeurosis palmar o fascitis palmar data de antiguo. En 1614 Félix Plater (1) invocó como agente causal a la luxación de los tendones flexores. Henry Cline (2) en 1808 ya describe someramente la contractura y en 1822 Sir Astley Cooper (3) explica el tratamiento mediante fasciotomía abierta. Sin embargo esta descripción fue superficial y hubo que esperar hasta el año 1832 en que el Baron Guillaume Dupuytren (4) hizo una descripción detallada, y aún vigente, de la enfermedad a la cual atribuyó un origen traumático y aplicó como tratamiento la fasciotomia abierta palmar. Son clásicas sus descripciones de los dos primeros enfermos operados un mayorista de vinos y un cochero. Desde entonces los artículos se han ido multiplicando y los autores intentan proporcionar una solución a ésta enfermedad de etiología desconocida

\section{Introducción}

En nuestro Servicio de Cirugía Plástica del Hospital del Sagrat Cor de Barcelona (España), venimos utilizando desde el año 1990, como tratamiento de la contractura de Dupuytren, básicamente la técnica de la palma abierta de Mcashe (5), aunque personalmente prefiero llamar a dicha técnica, de Dupuytren, ya que si leemos el trabajo original del año 1832, en él ya se mencionan los principios básicos de tratamiento quirúrgico de dicha enfermedad:

1. Incisiones transversales a nivel de los pliegues palmares.

2. Sección de los engrosamientos aponeuróticos responsables de las deformaciones.

3. Extensión de los dedos mediante un aparato las cuatro semanas que sigan a la operación.

4. Las heridas no son suturadas, permitiendo una cicatrización por granulación.

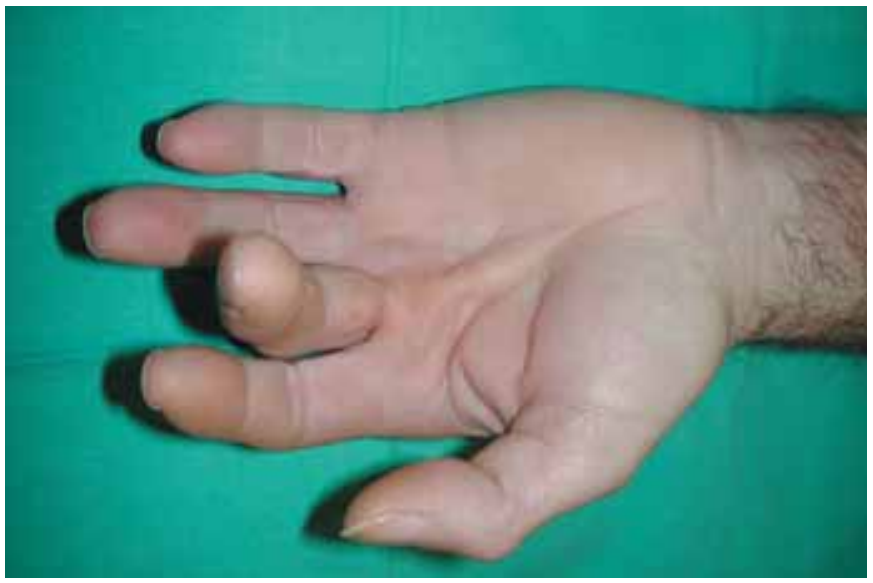

Tomando como punto de referencia las intervenciones efectuadas con anterioridad, es decir mediante la técnica de Mcashe (de Dupuytren), y los resultados obtenidos con la misma, observábamos secuelas postquirurgicas tales como: adherencias, atrofias cutáneas, hundimientos o depresiones de la superficie, parestesias o disestesias, dolor, etc., y también tomando como referencia la simple observación de la vida cotidiana y de la naturaleza, nos dimos cuenta de que siempre que se desea que algo no pase, no progrese, no avance, no evolucione y por lo tanto se detenga y se pare, hay que poner algún tipo de obstáculo que impida su avance; en este sentido basamos nuestra técnica.

\section{Material y Método}

Desde el año 2000 y durante un periodo de estudio de 42 meses, hemos intervenido un total de 44 pacientes afectos de la enfermedad de Dupuytren, a los cuales les hemos colocado un implante de silicona a nivel palmar.

Por sexos han sido 42 hombres y 2 mujeres con un total de 45 manos intervenidas (un paciente bilateral).

Las edades oscilan entre los 81 años el de mayor edad y 60 el de menor edad, obteniendo una media de 68 años en nuestra serie.

Con referencia a la lateralidad, 43 han sido manos derechas, 2 izquierdas y un caso de bilateralidad, siendo los dedos mas afectados el $3^{\circ}$ y el $4^{\circ}$. Al llegar a este punto hay que hacer unas puntualizaciones que tal vez sean las que hayan condicionado que los resultados sean enteramente satisfactorios en nuestra serie, como son: la homogeneidad del grupo tratado y los criterios de selección empleados, que consisten en: a) en ningún caso se ha incluido en nuestra serie una mano ya operada con anterioridad y b) el grado de afectación en todos los casos ha sido grado II y grado III. En nuestro medio no son intervenidos los grados 0 y I. No hemos tenido ningún caso de grado IV.

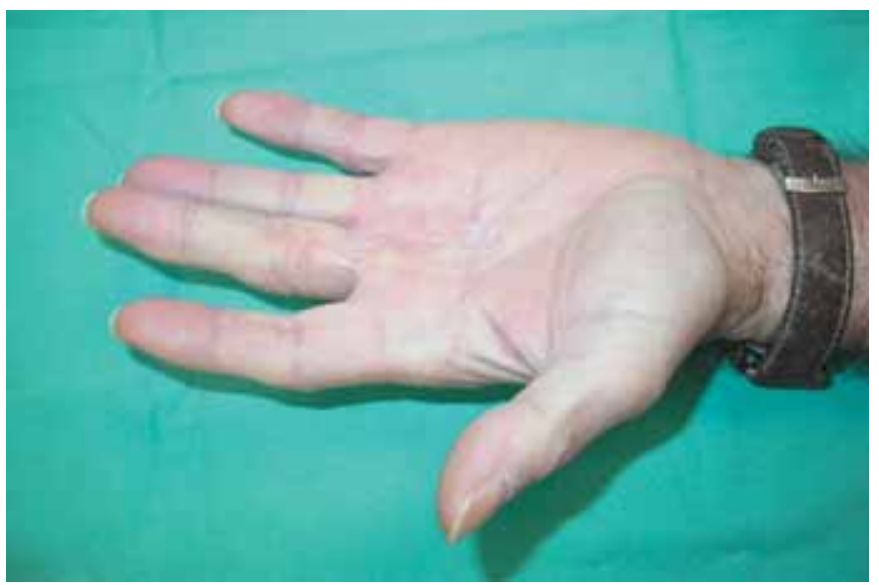

Fig. 1: a) Enfermedad de Dupuytren. Preoperatorio. b) Postoperatorio a los 18 meses. 


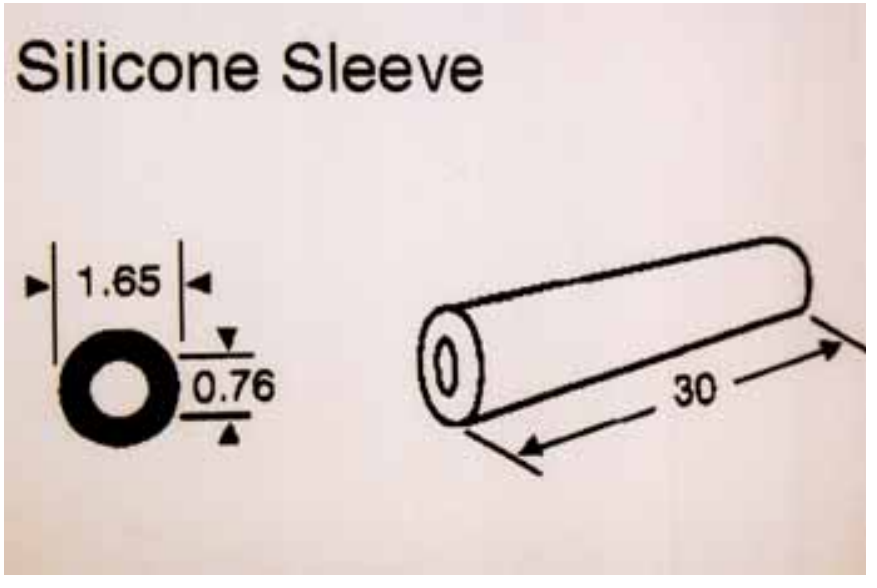

Fig. 2: Características del implante de silicona que utilizamos.

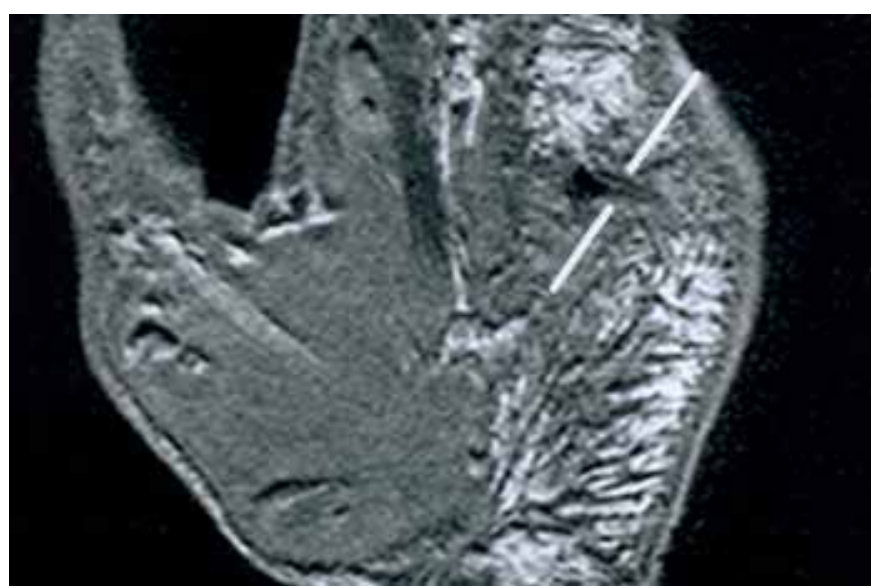

Fig. 4: RMN que demuestra el emplazamiento del implante de silicona.

En el tratamiento de la enfermedad de Dupuytren y concretamente en la afectación palmar (Fig. 1) de la misma, es donde hemos introducido una novedad que no está referida en la literatura médica mundial. Esta intervención se efectúa normalmente con una de las siguientes técnicas anestésicas: anestesia endovenosa, bloqueo nervioso del plexo axilar, anestesia general, que posibilitan la utilización de isquemia de la extremidad mediante manguito neumático.

La técnica quirúrgica consiste en efectuar las incisiones que se consideren necesarias a nivel palmar, utilizando preferentemente los pliegues palmares (mínimo uno, máximo tres). Posteriormente se practica la liberación cutánea, la identificación de las bandas aponeuróticas, con una meticulosa disección de las mismas para posteriormente realizar su exéresis parcial, consiguiendo que se produzca una solución de continuidad cómo mínimo de $1 \mathrm{~cm}$ entre las porciones proximal y distal de la banda aponeurótica (no se efectúa una eliminación completa de las mismas), preservando en lo posible las bandas transversales sobre todo a nivel palmar que tal como publico Skoog (6), nunca están afectadas y actuarán como soporte

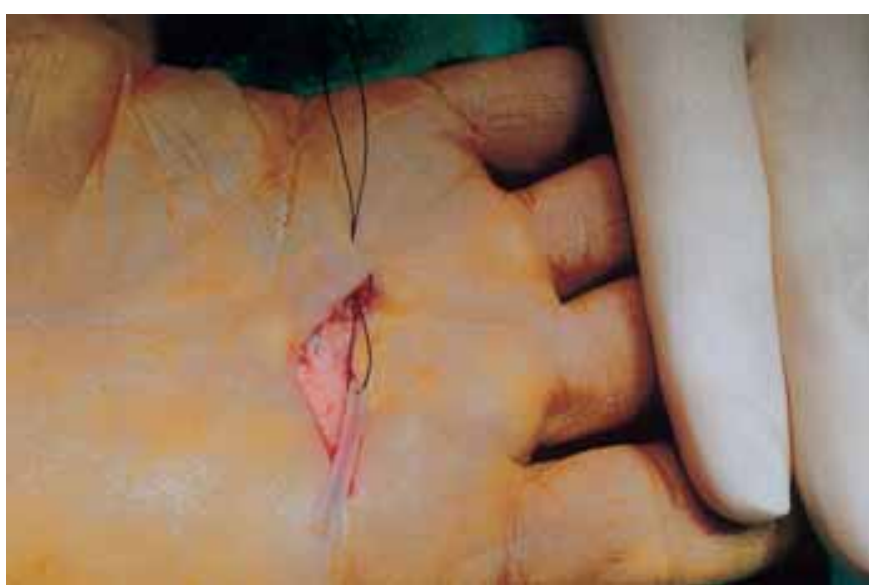

Fig. 3: Lugar donde normalmente se situa el implante.

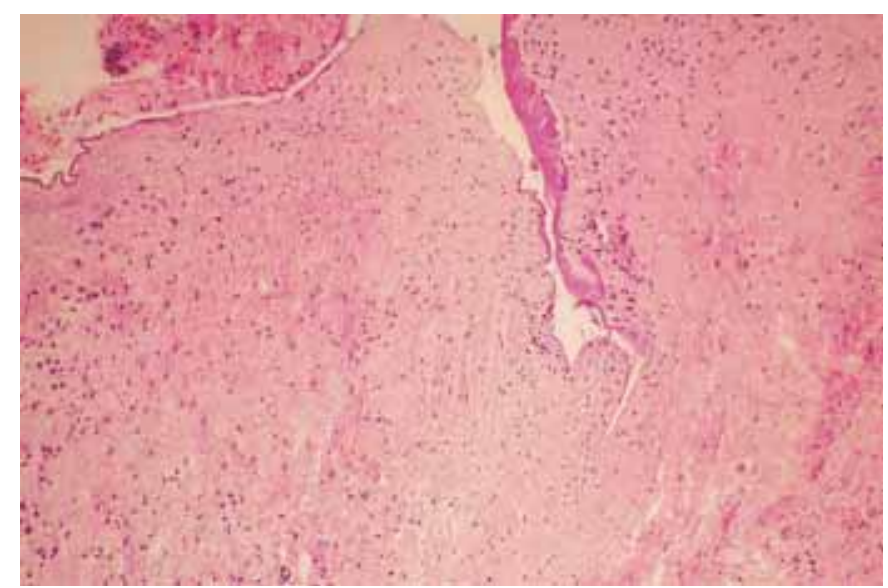

Fig. 5: Preparación histológica en la que se aprecia la fibrosis que se ha formado alrededor del implante (tinción E.0.50x).

anatómico de nuestro implante ; recurrimos durante la cirugía a la utilización de lentes de aumento con la finalidad de conseguir una disección, exéresis y hemostasia mucho más precisas.

Seguidamente pasamos a colocar nuestro implante, que consiste en un tubo hueco de silicona (Fig. 2 y 3 ): en el mercado disponemos de dos tamaños, uno que es de $1.85 \mathrm{~mm}$. de diámetro externo y de $0.76 \mathrm{~mm}$. de diámetro interno con $30 \mathrm{~mm}$. de longitud y otro de 2.4 $\mathrm{mm}$. de diámetro externo y $1.5 \mathrm{~mm}$. de diámetro interno y también de $30 \mathrm{~mm}$. de longitud. La utilización de dichos implantes se ha realizado únicamente en pacientes que no hayan manifestado o referido hipersensibilidad previa a polímeros de silicona.

La colocación se hace de forma que interrumpa la continuidad de la aponeurosis palmar; asimismo, no debe coincidir de manera perpendicular a la incisión efectuada para evitar un posible decúbito y extrusión del implante, y debe quedar cubierto y protegido por el tejido dermograso palmar. Posteriormente mediante sutura directa con Nylon monofilamento del 4/0 aseguramos la inmovilización y el aislamiento del mismo. 


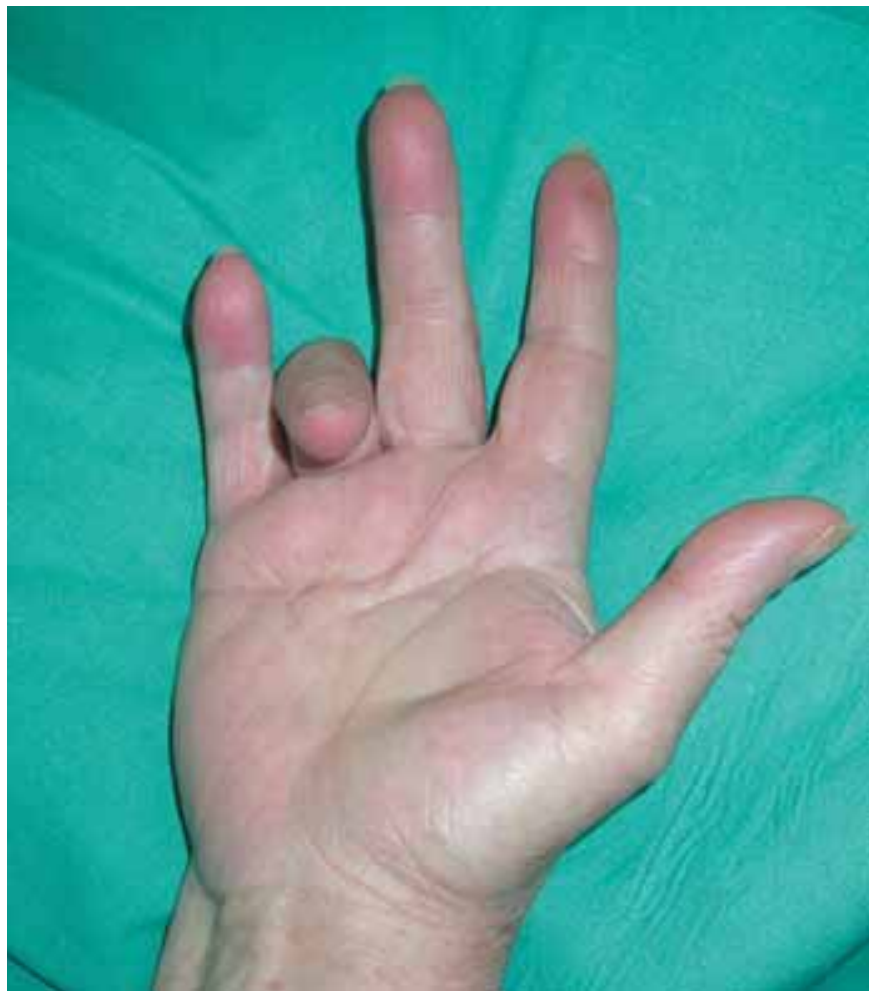

Fig. 6: a) Preoperatorio. b) Postoperatorio a los 12 meses.

Opcionalmente, en el caso de que debamos efectuar más de una incisión palmar, es imprescindible que la incisión en la cual se aloje y guarde el implante permanezca perfectamente suturada, pudiendo quedar las otras incisiones abiertas.

El tratamiento de la afectación digital se efectúa de

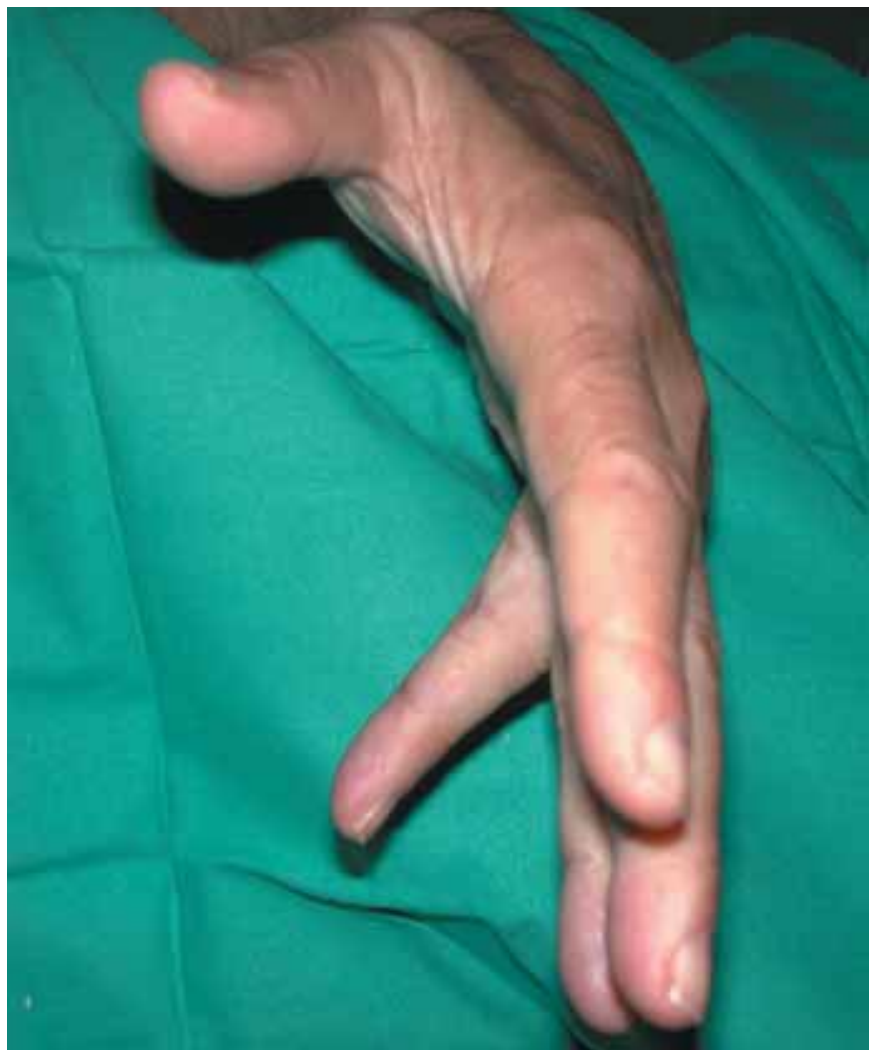

Fig. 7: a) Preoperatorio. b) Postoperatorio a los 18 meses.

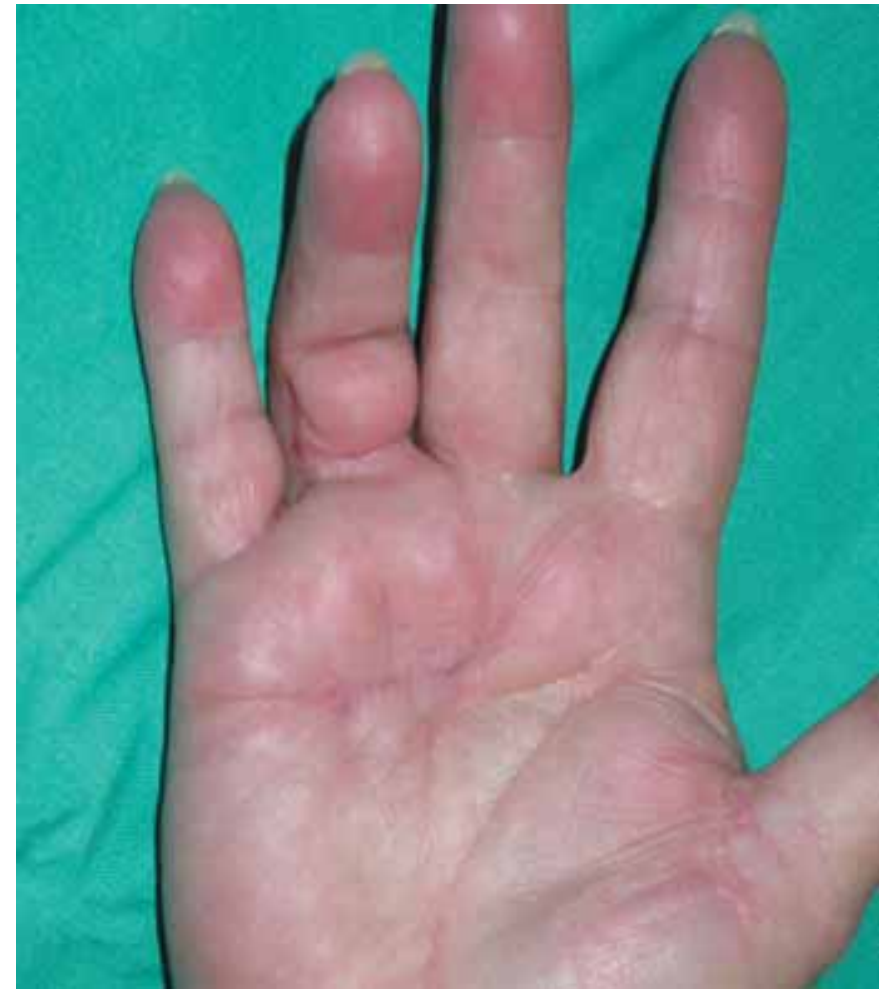

forma habitual, es decir mediante aponeurectomia parcial selectiva, sin colocar ningún tipo de implante en esta situación, o según la técnica mas pertinente en cada caso.

No dejamos ningún tipo de drenajes. Se efectúa seguidamente un vendaje compresivo con férula de extensión palmar y mantenimiento de la mano en elevación.

Podemos decir que en todos los casos excepto en uno en que se produjo la extrusión del implante, la tolerancia ha sido excelente; no se han producido signos inflamatorios o infecciosos en ningún caso (intraoperatoriamente se utiliza una dosis profiláctica de $1 \mathrm{gr}$ de Cefalosporina a dosis única); tampoco se ha

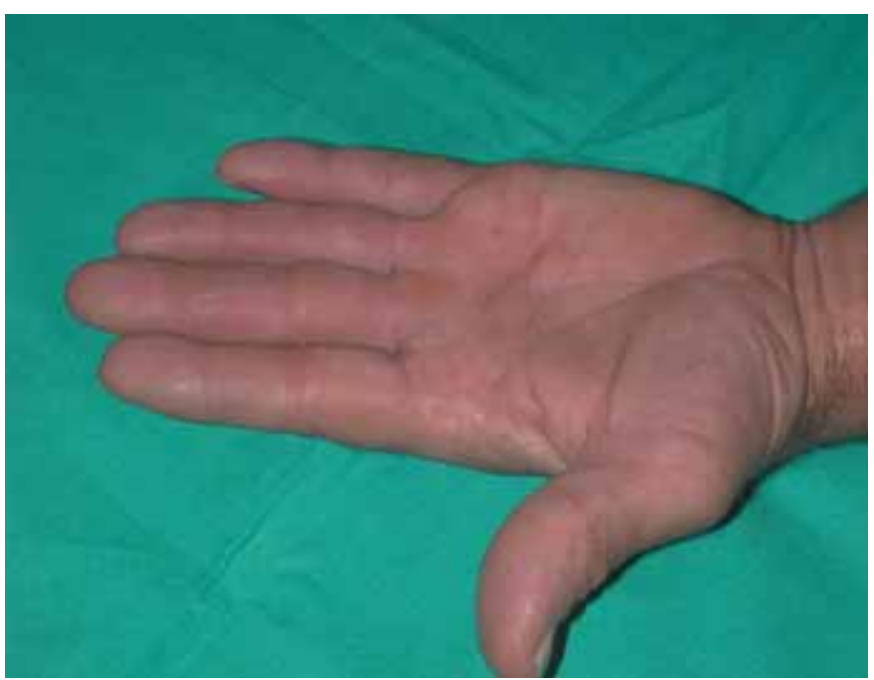




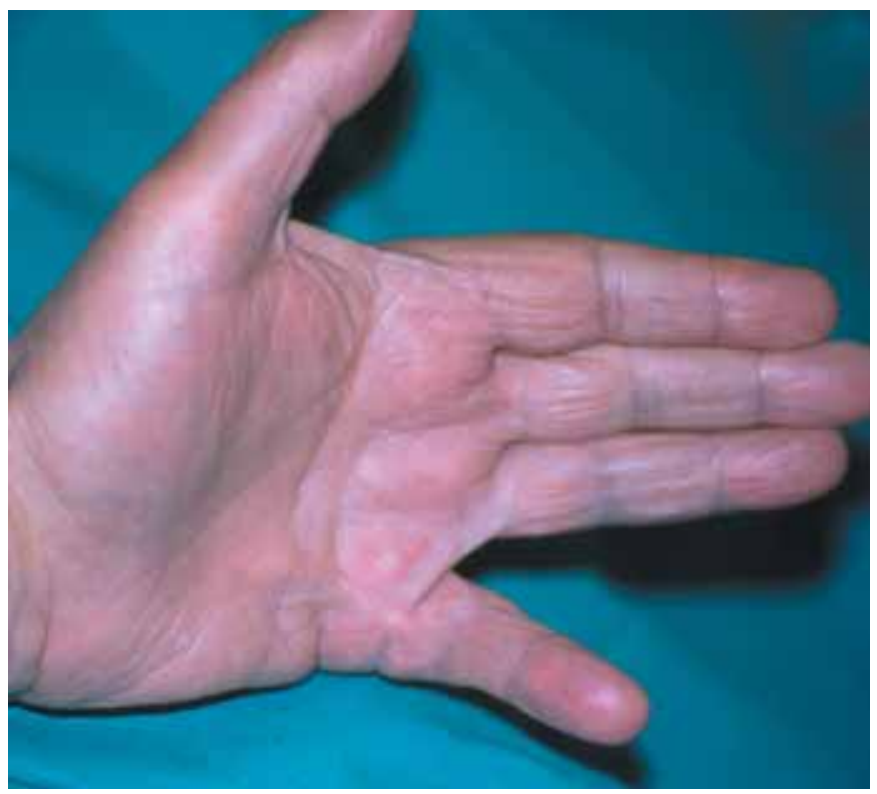

Fig. 8: a) Preoperatorio. b) Postoperatorio a los 12 meses.

constatado un aumento del dolor postoperatorio siendo similar al que se producía con la técnica clásica, precisando únicamente un tratamiento con Paracetamol oral a dosis de $500 \mathrm{mgr} \times 8 \mathrm{~h}$. El alta hospitalaria se produce a las 24 horas de la intervención previa revisión de las heridas; también se inicia un tratamiento de recuperación que efectúa el Servicio de Rehabilitación por espacio de un mes aproximadamente y el periodo de incapacidad ha sido idéntico al de la técnica clásica, alrededor de 45 días, momento en que se produce el alta definitiva.

En los casos que hemos ido controlando, y aunque el periodo de seguimiento de dicho procedimiento en el caso más antiguo es de 42 meses los pacientes no refieren ningún tipo de sintomatología y no se percibe a la palpación la existencia del implante, que es perfectamente tolerado.

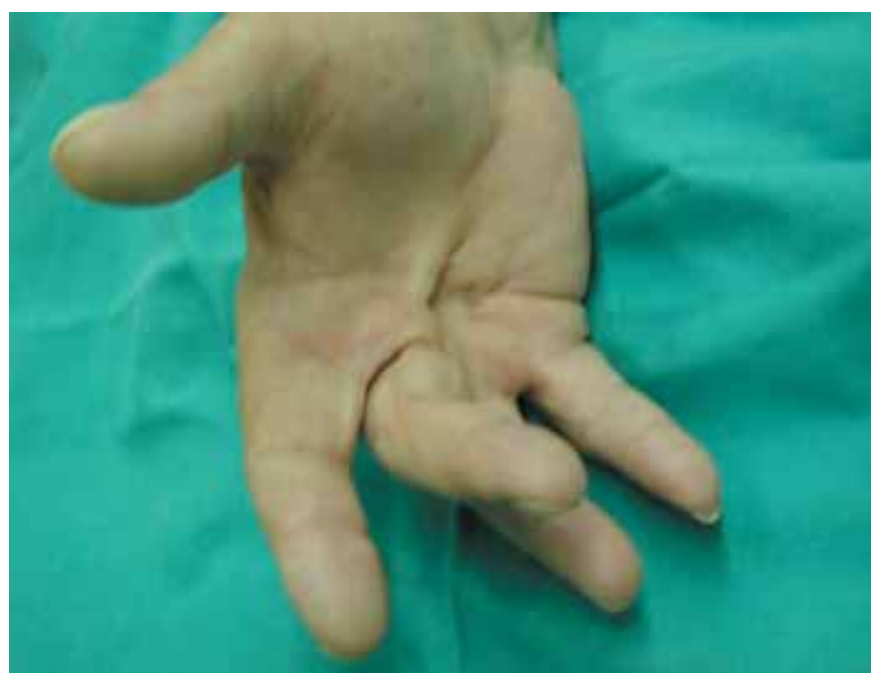

Fig. 9: a) Preoperatorio. b) Postoperatorio a los 3 meses.

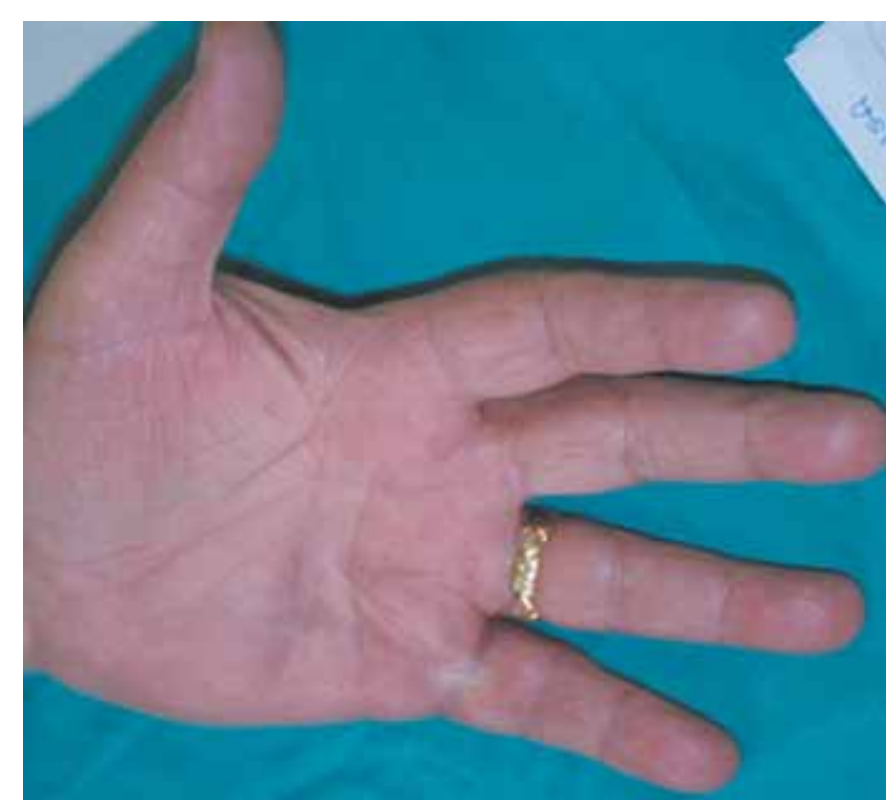

Resultados

Desde que iniciamos éste tipo de tratamiento y después de efectuar un total de 44 intervenciones quirúrgicas y de haber colocado 45 implantes, los resultados no pueden ser mas esperanzadores, puesto que tan sólo uno de los implantes se ha extruido (curva de aprendizaje, mala ubicación del mismo). El resultado funcional y estético ha sido excelente. Mostramos en la (Fig 4.) la imagen de un a Resonancia Nuclear Magnética (RNM) en la cual se demuestra la ubicación del implante de silicona, y la fibrosis que se instaura alrededor del mismo, lo que permite constatar el efecto esperado que es el de interrumpir, mediante un medio físico, la posible evolución de la enfermedad. En la figura 5 vemos la preparación histológica de un fragmento de implante que llevaba colocado 1 año y

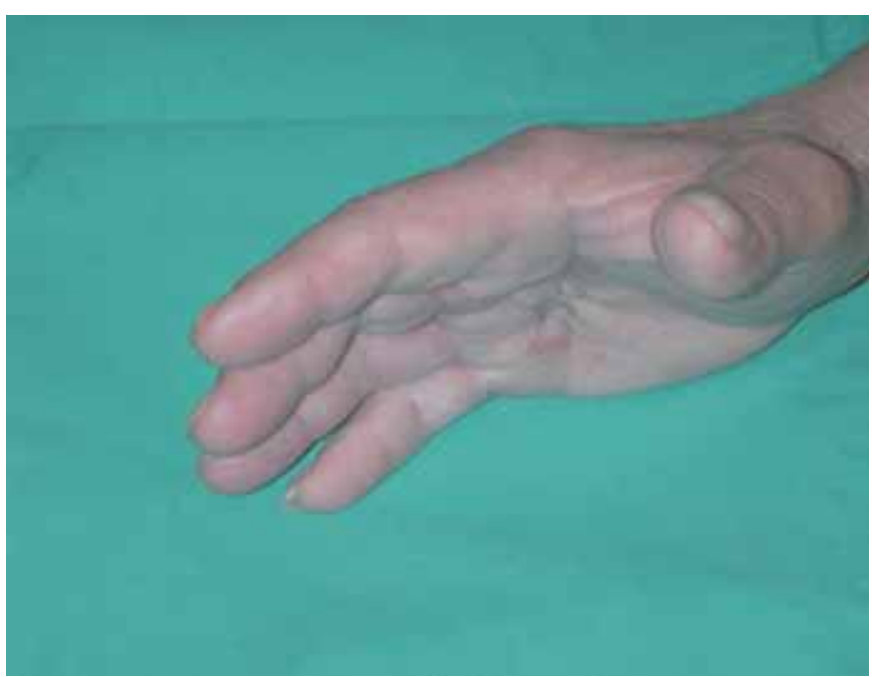




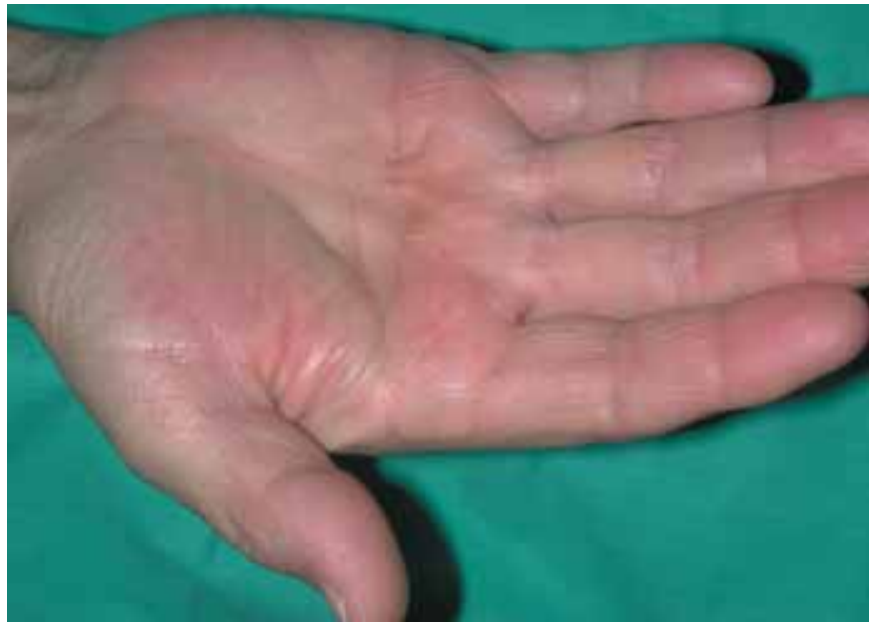

Fig. 10: a)Preoperatorio. b) Postoperatorio a los 6 meses.

podemos ver como, tanto alrededor del implante como dentro del mismo se ha formado un tejido fibroso a modo de cápsula periprotésica que actúa, según nuestra hipótesis de trabajo, como barrera natural (en el interior del tubo se observan reacción fibroblástica y pequeños vasos, compatible con la formación de tejido. Los estudios de investigación han demostrado que la cápsula, como estructura de colágeno, ya ha madurado a lo sumo a los dos meses de colocarse el implante y no experimenta cambios apreciables en los meses posteriores (7) (Fig. 6-10).

\section{Discusión}

La discusión en éste tipo de tratamiento podría situarse en los siguientes puntos:

1. Colocación de cuerpos extraños en la palma de la mano.

2. Reacción del organismo ante un implante.

3. Evolución a largo plazo.

4. Tolerancia del paciente.

5. Actividad laboral.

Después de efectuar una amplia revisión bibliográfica, no hemos encontrado en la bibliografía médica ningún apunte que haga referencia a la utilización de implantes (de cualquier tipo), y en concreto de silicona, como tratamiento de la contractura de la aponeurosis palmar en la enfermedad de Dupuytren.

La técnica quirúrgica que utilizamos para la colocación del implante no es complicada debiendo seguirse, según nuestra opinión, las recomendaciones anteriormente expuestas para conseguir una correcta ubicación del mismo que evite su posible extrusión y para que el implante consiga el efecto deseado, que es el actuar a modo de barrera que evite la progresión de la enfermedad, pero sobre todo, que se logre un resultado funcional excelente.

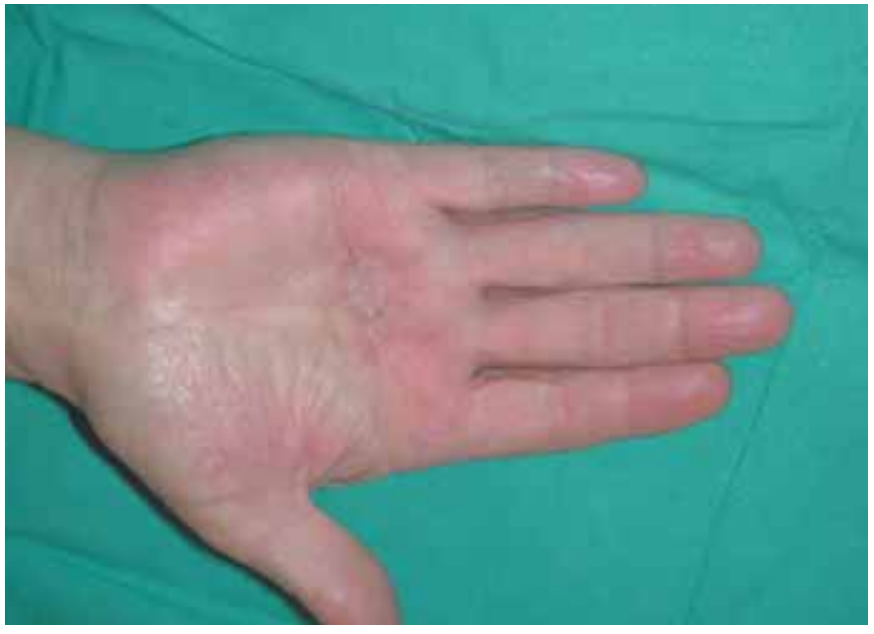

Por otra parte, el seguimiento efectuado a nuestros pacientes en el sentido de la tolerancia del implante demuestra que, todos refieren que no notan nada que les moleste o que les impida ejercer una función normal con la mano, siendo incluso prácticamente imposible el que puedan localizar la ubicación del mismo por palpación, lo que nos permite decir que, por el momento, la tolerancia es perfecta incluso en aquellos casos en que la actividad que se efectúa con las manos es importante (albañil, campesino, etc) (Fig. 5).

\section{Conclusiones}

Debido a que el periodo de seguimiento del procedimiento no es excesivamente largo, no podemos elevar y obtener conclusiones definitivas, pero si planteamos el tema en relación al tiempo que llevamos efectuando la técnica, puede catalogarse como segura y eficaz en aquellos pacientes que están referidos en nuestra serie. Solamente el tiempo nos podrá decir si será definitiva.

Si efectuamos a su vez una valoración de tipo económico cosa también importante, podemos decir que el costo de dicha intervención con nuestro procedimiento sólo se incrementa en 55 Euros.

\section{Agradecimientos}

A nuestros pacientes, por ellos, para ellos, con ellos. Gracias.

\section{Dirección del autor}

Dr. Juan Ballesta Alcaraz

Travesera de Dalt $\mathrm{n}^{\circ} 6$ - Entlo. E

08024 Barcelona (España)

e-mail: 14357jba@comb.es 


\section{Bibliografía}

1. Plater, F.: "Observationum, in hominis affectibus plerisque, corpi \& animo, funtionum laesione, dolore, aliave molestia \& vitio incomodantibus". C. Waldrick, Basileae 1614 p 140.

2. Cline, H.: Quoted by J. Windsor (1834). Lancet, 1808, 2; 501.

3. Cooper, A.: "A treatise on dislocations and Fractures". London. Longmans, Hurts, Rees, Dome and Browne. 1822; Pp. 524
4. Dupuytren, G.: « Leçons orales de clinique chirurgicale ». Published periodically by G. Bailliere, Paris 1833 .

5. McCash, CH, R.: "The open palm technique in Dupuytren's contracture". Br. J. Of Plast. Surg. 1964, 12: 271

6. Skoog,T.: "The transverse elements of the palmar aponeurosis in Dupuytren's contracture". Scand J Plast Surg 1967, 1:51.

7. Smahel, J. "Structure of capsules around silicone implants in hand surgery" The Hand, 1983, 15(1):47.

\section{Comentario al trabajo ıUtilización de implantes de silicona en la enfermedad de Dupuytrenı}

\begin{tabular}{c|c}
\hline Dr. Francisco Leyva Rodríguez \\
Médico Adjunto Servicio \\
de Cirugía Plástica, Estética, \\
Reparadora y Quemados \\
Hospital Universitario La Paz. Madrid
\end{tabular}

La enfermedad de Dupuytren fué descrita por el barón de Dupuytren en 1831en su cochero al que le realizó una fasciotomia. Esta enfermedad afecta principalmente a escandinavos y descendientes del oeste de Europa. La enfermedad de Dupuytren es el resultado del engrosamiento y contractura de la fascia palmar y finalmente de la fascia digital como consecuencia de la hiperplasia de fibroblastos y miofibroblastos y la secrección excesiva de colágeno tipo III. La fasciectomía selectiva es el método de tratamiento más usado.

Por otra parte, en determinados pacientes, habitualmente ancianos, con patología asociada y con la enfermedad localizada se pueden usar técnicas no quirúrgicas o mínimamente invasivas. De este grupo de técnicas, las que más se han usado aunque generalmente con resultados modestos son las fasciotomias con bisturí o con aguja, la tracción esquelética lenta y continua, la radiación, el dimetil-sulfóxido, la vitamina E, el alopurinol, la terapia física, los ultrasonidos, los estroides, el interferon, las férulas, las enzimas como las colagenasas, el 5-fluoruracilo, el tamoxifeno, la sinvastatina o el iminoqued como más estudiados(1).

El fin último de ambos tipos de tratamientos es mejorar la función de la mano y prevenir la recidiva, ya que ésta es una de las evoluciones más temidas por cualquier cirujano de mano.

Este trabajo supone un empeño loable en buscar soluciones a un problema complejo y al que probablemente por su escasa repercusión social se le presta poca atención como es la enfermedad de Dupuytren. Tras la lectura del trabajo me surgen algunas preguntas.

Se realiza a todos los enfermos, por tanto pretende desplazar a la fasciectomía selectiva tradicional. Parece que la ventaja inicial de esta técnica es evitar la fasciectomia selectiva palmar, que no la del dedo; sin embargo la cirugía de la enfermedad en los dedos es la más difícil y la que más complicaciones iatrogénicas nos puede deparar, y esta técnica no lo evita.

Si se indicara solo en casos de afectación palmar o en pacientes que no pueden ser sometidos a cirugía más agresiva la aponeurotomía (2) y aún mejor la aponeurectomia parcial de la brida parece ofrecer buenos resultados como señalan otros autores como Moermans(3) y Clibbon (4). Si se quiere introducir una mejora implantando material de interposición se deberá comparar con las técnicas que no introducen nada. No hay que olvidar lo que supone la colocación de un material extraño en la palma de la mano y aunque la literatura médica en este campo ofrece resultados variables parece arriesgado interponer un material extraño para comprobar una hipótesis como la de la interrupción u obstáculo máxime cuando otros autores como hemos dicho antes consiguen lo mismo sin colocar materiales extraños.

Igualmente no me parece válido desde un punto de vista científico señalar que los resultados son excelentes si no hay unos parámetros objetivables que permitan identificar cada paciente con sus características pre y postoperatorias.

No sabemos si hay recidiva de la enfermedad o en qué grado, pues no se menciona y ese es el fin último de la cirugía, además de que el tiempo medio de estudio es breve.

La discusión no me parece que ayude pues creo que son más bien las conclusiones extendidas, ya que en ningún momento se discute la necesidad de hacer esta técnica, la metodología, los problemas de introducir materiales extraños o de por qué no están descritos previamente, qué ventajas aporta frente a otras.

Por todo ello considero que a pesar de mostrar un trabajo entusiasta y con ideas novedosas parece poco probable convencer a la mayoría de los cirujanos de que introduzcan en la palma de la mano un cuerpo 
extraño, a pesar del buen resultado obtenido por los autores.

\section{Bibliografía}

1. Hurst LC, Badalamente MA.: "Nonoperative treatment of Dupuytren's disease". Hand Clin 1999; 15: 97.
2. Foucher G, Medina J, Navarro R.: "Percutaneous needle aponeurotomy: complications and results". J Hand Surg [Br]. 2003; 28: 427.

3. Moermans JP.: "Long-term results after segmental aponerectomy for Dupuytren's disease”. J Hand Surg 1996; 21B: 797.

4. Clibbon JJ, Logan M.: "Palmar segmental aponeurectomy for Dupuytren's disease with metacarpophalangeal flexion contractura”. J Hand Surg (Br) 2001; 26B: 360.

\section{Respuesta al comentario del Dr. F. Leyva}

Agradezco ante todo el comentario efectuado por el Dr. Leyva Rodríguez, sobre el trabajo, además si proviene de un grupo de trabajo tan reconocido como el Hospital Universitario La Paz de Madrid. Gracias.

Todas y cada una de las apreciaciones que se efectúan son ciertas en el contenido académico y me muestro de acuerdo con ellas en un porcentaje muy alto Hasta aquí podría concluir gracias por vuestra inestimable colaboración punto y final, pero eso no seria correcto.

Quiero puntualizar algo que para mí es importante y diría que crucial. No estamos delante de un trabajo comparativo, ni delante de un trabajo retrospectivo, ni delante de un trabajo epidemiológico. Estamos delante de un trabajo original e inédito; se presentó un trabajo preliminar en el congreso de la SECPRE de Oviedo del año 2002. Es una técnica que se puede efectuar en los casos de Enfermedad de Dupuytren, independiente del grado que tengan y que únicamente hemos introducido la premisa de que deben tratarse pacientes que no hayan sido intervenidos con anterioridad. Quedan por consiguiente excluidas las recidivas para evitar introducir variables nuevas que pudieran interferir en la valoración de resultados, y que sigue escrupulosamente todos los postulados de tratamiento de dicha enfermedad tal como fueron descritos por el barón Guillaume de Dupuytren en el año 1831.

Quiero comentarle a Ud. Dr.Leyva que no se trata de una técnica no quirúrgica o mínimamente invasiva, ni tampoco se trata de una técnica "médica". Es una técnica quirúrgica. Técnica quirúrgica que no evita la fasciectomia selectiva palmar ni tampoco la del dedo. Estoy de acuerdo que sería bueno comparar resultados con otras técnicas, para hacerlo de una manera cierta al $100 \%$ se tendría que efectuar con pacientes afectos de Dupuytren bilateral y con el mismo grado de afectación, en los cuales efectuaríamos una técnica diferente en cada una de las manos. A nadie se le escapa que esto es prácticamente imposible.

A nadie se le escapa tampoco que los materiales extraños en cualquier parte del organismo suelen ser una "fuente" de problemas, pero sí que puedo intentar transmitirle mi experiencia personal, no lo que dice la bibliografía médica y lo que dicen otros autores, normalmente anglosajones por supuesto respetables y válidos, únicamente he tenido un problema y fue en el primer caso que

\section{Dr. J . Ballesta Alcaraz}

realizamos; a partir de este punto de inflexión y que atribuyo a la curva de aprendizaje, no he tenido ningún otro problema hasta la fecha presente, y todos los pacientes han seguido un exhaustivo seguimiento y control.

Estará Ud. de acuerdo conmigo en que muchas veces, sobre todo a nivel palmar, se producen secuelas postquirúrgicas tales como atrofia cutánea, atrofia subcutánea desaparición o disminución del tejido graso, adherencias, disestesias, parestesias etc.; con nuestra técnica esto ha desaparecido y el implante es tolerado e imperceptible en todos los casos. El implante que se utiliza, no es un implante cualquiera ni un implante fruto de la casualidad, es un implante que se ha diseñado para obtener un efecto deseado que es el de "fibrosis dirigida " $\mathrm{o}$ " fibrosis orientada" cuyo objetivo es cortar la normal evolución de la enfermedad, concepto este también nuevo

Es por otra parte evidente que la casuística que se presenta no es grande, pero si lo suficiente para considerarse significativa y el hecho de señalar que los resultados son excelentes solo viene a indicar que comparando los resultados por mí obtenidos con las técnicas clásicas, con los actuales utilizando implantes de silicona, han mejorado de una forma evidente, término este poco científico pero real. Soy consciente de que una casuística mayor, un seguimiento en el tiempo más largo y una valoración del índice de recidivas (término este diferente al de progresión de la enfermedad) y que la misma técnica sea efectuada por diferentes cirujanos, serán los que darán la validez a ésta técnica.

Le agradezco sus calificativos de entusiasta y novedoso, son muy amables, de eso se trata, simplemente hay que ver la bibliografía que Ud. Dr. Leyva aporta y que en ningún caso hace referencia a la utilización de implantes para el tratamiento de dicha enfermedad ¿no ha encontrado citas bibliografiítas de referencia en tal sentido?.

Disculpe que yo no pueda aportar ninguna bibliografía consultada pero es que referente a éste tema no existe. Tampoco es mi objetivo el crear una secta de cirujanos adictos a los implantes de silicona para el tratamiento de dicha enfermedad, ni tampoco los tengo patentados ni registrados, ni llevan mi nombre, simplemente expongo una técnica quirúrgica personal, por si puede resultarle útil a algún colega.

Saludos. 